(C) 2021, The Authors. Published by Elsevier Inc. and Fass Inc. on behalf of the American Dairy Science Association ${ }^{\circledR}$. This is an open access article under the CC BY-NC-ND license (http://creativecommons.org/licenses/by-nc-nd/4.0/).

\title{
Vitamin E analogs limit in vitro oxidant damage to bovine mammary endothelial cells
}

\author{
M. J. Kuhn (1) and L. M. Sordillo* (1) \\ Department of Large Animal Clinical Sciences, College of Veterinary Medicine, Michigan State University, East Lansing 48824
}

\begin{abstract}
Diseases that occur during the transition period are exacerbated when cows are unable to cope with an increased pro-oxidant load that results in oxidative stress. Dairy cattle are routinely supplemented with the vitamin $\mathrm{E}$ analog $\alpha$-tocopherol to mitigate the severity of oxidative stress. Nonetheless, oxidative stress remains a disease predisposing condition for many dairy cattle. A better method of optimizing the antioxidant functions of vitamin $\mathrm{E}$ is needed. $\alpha$-Tocopherol is only 1 of 8 analogs of vitamin E, all of which have varying antioxidant properties in other mammals, albeit a shorter physiological half-life compared with $\alpha$-tocopherol. A primary bovine mammary endothelial cell oxidant challenge model was used to determine functions of certain vitamin $\mathrm{E}$ analogs. The aim of this study was to determine if other analogs, namely $\gamma$-tocopherol or $\gamma$-tocotrienol, have antioxidative functions in bovine cells and if these functions may protect cellular viability and endothelial function from oxidant damage. Physiological (10 $\mu M)$ and supraphysiological $(50 \mu M)$ concentrations of $\gamma$-tocopherol and $\gamma$-tocotrienol had a greater capacity to reduce accumulated reactive oxygen species derived from a nitric oxide donating pro-oxidant antagonist, when compared with $\alpha$-tocopherol, after 30 min to $6 \mathrm{~h}$ of treatment. Further, $\gamma$-tocotrienol $(10 \mu M)$ decreased cell cytotoxicity to a greater amount than other analogs at like concentrations, whereas $\gamma$-tocopherol $(10 \mu M)$ reduced lipid peroxidation and apoptosis more effectively than other analogs. Last, $\alpha$-tocopherol (5 and $10 \mu M$ ) and $\gamma$-tocopherol (5 and $10 \mu M)$ significantly slowed pro-oxidant induced loss of endothelial cell barrier integrity over a 48-h period using an electrical cell-substrate impedance sensing system. Concerningly, $\gamma$-tocotrienol drastically reduced the endothelial barrier integrity at only $5 \mu M$ despite no apparent effect on
\end{abstract}

Received September 20, 2020.

Accepted February 9, 2021.

*Corresponding author: sordillo@msu.edu cellular viability at like concentrations. $\gamma$-Tocotrienol, however, was also the only analog to show significant cytotoxicity and reductions in viability at supraphysiological doses $(25$ and $50 \mu M)$. Our results suggest that $\gamma$-tocopherol has antioxidant activities that reduces cellular damage and loss of function due to oxidant challenge as effectively as $\alpha$-tocopherol. These data set the foundation for further investigation into the antioxidant properties of vitamin $\mathrm{E}$ analogs in other bovine cells types or whole animal models.

Key words: vitamin E, dairy cattle, oxidative stress, tocopherol, tocotrienol

\section{INTRODUCTION}

Dairy cattle have an increased susceptibility to health disorders during the transition period. The progressive development of oxidative stress during the transition from late gestation to peak lactation is thought to be a significant underlying factor leading to increased incidence and severity of disease (Sordillo and Mavangira, 2014). Oxidative stress results when there is an imbalance between increased production of reactive oxygen species (ROS) and the availability of antioxidant defenses needed to reduce ROS accumulation that results in damage to macromolecules such as lipids, proteins, and DNA. Indeed, oxidative stress can contribute to immune and inflammatory dysfunction around the time of calving, thus enhancing the occurrence of infectious and metabolic diseases (Celi, 2011; Mavangira and Sordillo, 2018).

Vitamins with antioxidant functions are used widely by the dairy industry to increase the antioxidant capacity of animals and promote a regulated oxidant environment. Vitamin $\mathrm{E}$ is one such vitamin with antioxidant functions and has been increasingly supplemented over the past $30 \mathrm{yr}$ in its most bioactive form, $\alpha$-tocopherol (Weiss, 1998, 2017). Despite doubling circulating concentrations of $\alpha$-tocopherol over the last 3 decades, some dairy cattle still face oxidative stress around the time of calving (Kuhn et al., 2018). Although it may be intuitive to supplement greater concentrations of $\alpha$-tocopherol, further increases in supplementation 
above current industry standards have yielded concerning results. In 1 study, supplementing $\alpha$-tocopherol at 3 times National Research Council recommendations resulted in increased incidence of disease and an increased production of reactive oxygen metabolites in certain groups of animals (NRC, 2001; Bouwstra et al., 2010a, 2010b). With a need for increased antioxidant capacity and reluctance to supplement greater amounts of $\alpha$-tocopherol, there is a considerable need for a new approach to antioxidant supplementation in transition dairy cattle.

Although $\alpha$-tocopherol is found at the greatest concentrations of any analog in mammals, it is only 1 of 8 vitamin $\mathrm{E}$ analogs, all of which retain certain functions similar to $\alpha$-tocopherol such as antioxidant activities (Jiang, 2014). Analogs differ based upon the methylation of their chromanol ring, resulting in $\alpha$, $\beta, \gamma$, and $\delta$ configurations and a saturated (tocopherols) or unsaturated (tocotrienols) phytyl tail (Figure 1). These slight differences in configuration result in significantly different rates of metabolism, generally following a pattern of $\gamma>\delta>\beta>\alpha$ and tocotrienols $>$ tocopherols (Sontag and Parker, 2007). Additionally, analogs without a methyl group at the 5 position of the chromanol ring, such as those designated with $\gamma$ and $\delta$ configurations, have a greater capacity to reduce reactive nitrogen species (Cooney et al., 1993). For these reasons, it is possible that non- $\alpha$-tocopherol analogs of vitamin $\mathrm{E}$ may contribute to the overall antioxidant
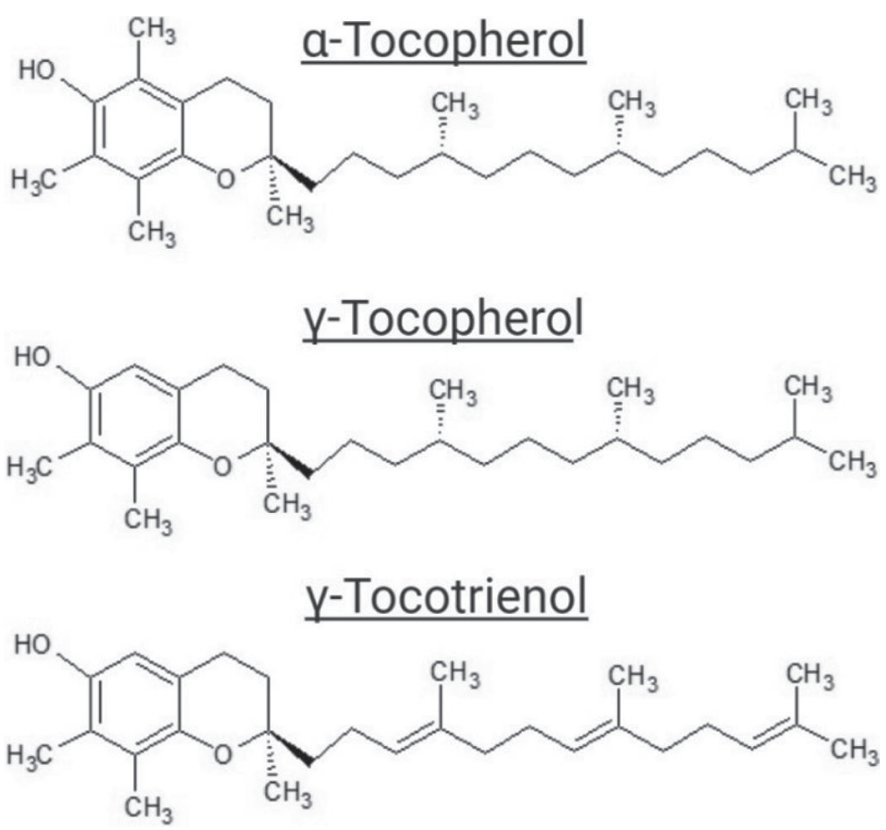

Figure 1. Chemical structures of the 3 analogs used for the current study; namely, $\alpha$-tocopherol, $\gamma$-tocopherol, and $\gamma$-tocotrienol. Figure created with ChemSketch and BioRender.com. capacity of an animal with a reduced risk of adverse effects due to faster metabolism.

Despite examples of antioxidative function in other species, research into non- $\alpha$-tocopherol analog bioactivity and potential use in dairy cattle is sparse. Mixed tocopherol supplements have been fed to calves and mid-lactation cattle without adverse effects; however, tocotrienols are yet to be used in a similar manner, leaving their safety and efficacy in cattle unknown (Elsasser et al., 2013; Qu et al., 2018). Promisingly, calves fed a tocopherol mix had reduced nitration of tyrosine, a marker of reactive nitrogen species production, in liver biopsy samples after intravenous challenge with LPS compared with control calves. The reduction in tyrosine nitration conferred by $\gamma$-tocopherol was comparable to those fed strictly $\alpha$-tocopherol, suggesting that a non- $\alpha$-tocopherol mix can protect tissue from damage equally well as $\alpha$-tocopherol (Elsasser et al., 2013). Unfortunately, this study in calves and other studies focusing on the effects of mixed tocopherol supplementation on adult cattle do not evaluate redox balance or measure oxidative stress in any other way, leaving a significant gap in knowledge as to the antioxidant properties of vitamin $\mathrm{E}$ analogs in cattle (Borher et al., 2002; Elsasser et al., 2013; Qu et al., 2018).

The aim of this study was to assess the capacity of vitamin $\mathrm{E}$ analogs $\gamma$-tocopherol and $\gamma$-tocotrienol in comparison to $\alpha$-tocopherol to limit cellular damage, death, and loss of function in an in vitro oxidative stress model. In vivo, concentrations of blood $\alpha$-tocopherol and $\gamma$-tocopherol can reach concentrations after supplementation $>35 \mu M$ and $7 \mu M$, respectively, making concentrations evaluated herein comparable to those found in dairy cattle (Qu et al., 2018). Unfortunately, supplementation of $\gamma$-tocotrienol has not been reported in cattle, although physiological values would be expected to be lower than concentrations used in this study due to rapid metabolism. Using primary bovine mammary endothelial cells (BMEC), we first assessed the antioxidant capacity of vitamin $\mathrm{E}$ analogs and their potential cytotoxicity to cells. Having established these roles, we further characterized the effects of increased antioxidant capacity on cell health, evaluating oxidatively induced cytotoxicity and apoptosis. Finally, endothelial cell barrier integrity was observed to understand the effect of vitamin $\mathrm{E}$ analogs on endothelial barrier integrity and function when oxidatively challenged.

\section{MATERIALS AND METHODS}

\section{Chemicals and Reagents}

Vitamin E analogs were obtained from Cerillant Corp. ( $\alpha$-tocopherol and $\gamma$-tocopherol) or Cayman 
Chemical ( $\gamma$-tocotrienol). YoPro and propidium iodide (PI) dyes were purchased from Invitrogen, and (Z)-1-[N-(2-aminoethyl)- $N$-(2-ammonioethyl)amino] diazen-1-ium-1,2-diolate (DetaNO) was purchased from Cayman Chemical Co. Antibiotic/antimycotic, trypsin $(0.05 \%)$, glutamine, and bovine collagen were obtained from Life Technologies. Fetal bovine serum (FBS) was procured from Hyclone Laboratories Inc., and HEPES buffer and Ham's F12K from Corning Inc. Hanks' balanced salt solution (HBSS) was purchased from Sigma, and gentamycin and collagenase were purchased from Gibco.

\section{Primary Cell Isolation and Culture}

Primary BMEC were isolated as described previously (Aherne et al., 1995). In short, pudendal arteries were obtained from an abattoir and placed in ice-cold HBSS with $0.1 \%$ gentamycin for transportation to the laboratory. Once at the laboratory, vessels were rinsed with HBSS, clamped at one end, and filled with collagenase solution (100 mg of collagenase in $48 \mathrm{~mL}$ of KrebsRinger bicarbonate and $2 \mathrm{~mL}$ of $7.5 \% \mathrm{BSA}$ ). Once filled, the open end was clamped shut, and vessels were incubated at $37^{\circ} \mathrm{C}$ for $10 \mathrm{~min}$. After incubation, collagenase solution was collected, and vessels were rinsed with rinse liquid collected into the same tube as the collagenase solution. Cells were pelleted, resuspended in Ham's F-12K growth medium containing 10\% FBS, 10 $\mathrm{m} M$ HEPES buffer, $0.25 \%$ sodium bicarbonate, $2 \mathrm{mM}$ L-glutamine, 1\% 1:1 antibiotic:antimycotic, $100 \mu \mathrm{g} / \mathrm{mL}$ heparin, $10 \mu \mathrm{g} / \mathrm{mL}$ insulin, $5 \mu \mathrm{g} / \mathrm{mL}$ transferrin, and $40 \mathrm{ng} / \mathrm{mL}$ sodium selenite. This is the base medium on which all other treatment media were based, differing in the amount of FBS they contained, which will be signified by the initial percentage and the presence or absence of sodium selenite, represented as + Se or - Se. With this understanding, the growth medium will be referred to as $10 \% \mathrm{~F} 12 \mathrm{~K}+$ Se medium. This resuspension of cells was then transferred to a T25 culture flask. After reaching confluency, cells were trypsinized for collection and serially diluted for plating on 96-well plates for single-cell isolation. Wells growing colonies from single endothelial cells were propagated before freezing in liquid nitrogen and eventual use. Cells were thawed and used at passages 6 to 10 for assays.

\section{Cell Viability}

Cell viability was measured using the Cell Titer-Glo (Promega) assay. The Cell Titer-Glo assay uses cellular ATP released after cell lysis to drive a luciferase reaction that results in a quantifiable luminescent com- pound. The amount of ATP available for this reaction is reflective of the number of viable cells in each sample. Cells were plated in triplicate in white flat-bottom 96well plates and allowed to incubate overnight in $10 \%$ F12K +Se medium. Six hours before treatment, the medium was replaced, and for those receiving a vitamin $\mathrm{E}$ analog or vehicle control, this was included. For all assays, vehicle control is equivalent to the greatest amount of methanol that would be used for delivery of a vitamin $\mathrm{E}$ analog in that particular assay. At the treatment time point, the medium was replaced with $0 \% \mathrm{~F} 12 \mathrm{~K}$-Se medium containing 0.25 to $5 \mathrm{mM}$ of DetaNO antagonist, 10,25 , or $50 \mu M$ of vitamin $\mathrm{E}$ analog, or methanol vehicle control, depending on the assay. A set of wells received the medium only as a control, and a set of wells that did not contain cells received the medium only to record background luminescence for each plate as well. Plates were incubated at $37^{\circ} \mathrm{C} 5 \%$ $\mathrm{CO}_{2}$ for the designated time, after which manufacturer instructions were followed for measurement of ATP. In short, $100 \mu \mathrm{L}$ of Cell Titer-Glo reagent was added to the wells, mixed for 2 min on a shaker plate, incubated for $10 \mathrm{~min}$ at room temperature, and read for luminescence by a BioTek Synergy H1 microplate reader.

Analog cytotoxicity was quantified by lactate dehydrogenase (LDH) release with a CyQUANT LDH release assay (Invitrogen). The CyQUANT LDH release assay uses LDH released from cells caused by cellular damage or death as a catalyst for a lactate-to-pyruvate reaction that, in turn, reduces $\mathrm{NAD}^{+}$to NADH. Subsequently, NADH is used to produce a quantifiable color shift by reducing tetrazolium salt to red formazan that is proportional to cell cytotoxicity. Cells were plated in triplicate in clear, flat-bottomed 96-well plates and allowed to incubate overnight in $10 \% \mathrm{~F} 12 \mathrm{~K}+$ Se medium. Six hours before treatment, the medium was replaced, and for those receiving a vitamin $\mathrm{E}$ analog or vehicle control, this was included. The 2 experiments evaluating cytotoxicity diverge in method at this point. For the evaluation of vitamin $\mathrm{E}$ analog cytotoxicity, at the treatment time point the, medium was replaced with a $0 \% \mathrm{~F} 12 \mathrm{~K}$-Se medium containing vitamin $\mathrm{E}$ analogs at concentrations of 10,25 , or $50 \mu M$ or methanol vehicle control for appropriate wells. For assessing the capacity for vitamin $\mathrm{E}$ analogs to prevent BMEC cytotoxicity from the antagonist, at treatment time cells were treated with $2 \mathrm{mM}$ DetaNO and with $10 \mu \mathrm{M}$ of individual analogs, a mixture of $10 \mu M$ of each analog, or $30 \mu M$ of $\alpha$-tocopherol alone. For all cytotoxicity assays, a set of wells received medium only as a control considered to have $0 \%$ cytotoxicity and a set of wells received a lysing buffer after incubation that was considered to have $100 \%$ cytotoxicity. An additional set of wells that did 
not contain cells received medium only to record background absorbance, and a further triplicate received a positive control mixture from the manufacturer that was reliably positive. Plates were incubated at $37^{\circ} \mathrm{C}$ in $5 \% \mathrm{CO}_{2}$ for the designated time, after which time manufacturer instructions were followed for measurement of LDH release. In short, after incubation, wells that had contained $150 \mu \mathrm{L}$ of medium received either $15 \mu \mathrm{L}$ of Triton X-100 lysing buffer or ultrapure water and were incubated at room temperature for $5 \mathrm{~min}$, after which $90 \mu \mathrm{L}$ was transferred to a new identical plate. Ten microliters of assay reagent was added to all wells, and the plate was incubated at $37^{\circ} \mathrm{C}$ in $5 \%$ $\mathrm{CO}_{2}$ for $30 \mathrm{~min}$, after which absorbance was measured. Colorimetric readings were taken on a BioTek Synergy H1 microplate reader. Data are represented as the percentage of cytotoxicity based upon maximum and minimum LDH release.

\section{Reactive Metabolite Quantification}

Reactive oxygen species were quantified with the OxiSelect intracellular ROS assay (Cell Biolabs) by reaction of $2^{\prime}, 7^{\prime}$-dichlorodihydrofluorescein dye with ROS, creating fluorescent dichlorodihydrofluorescein that can be quantified. Cells were plated in black-walled, flat, clear-bottom 96-well plates in triplicate with wells left empty aside from medium to measure background fluorescence. After incubation overnight, the medium was replaced with like $10 \%$ F12K +Se medium only for DetaNO titration or with vitamin $\mathrm{E}$ analogs or vehicle control, as applicable, for assessment of the ability of the vitamin $\mathrm{E}$ analogs to mitigate ROS production, and incubated for $6 \mathrm{~h}$. After this incubation, cells were washed twice with warm HBSS and loaded with a 1:200 dilution of the cell-permeable fluorogenic probe $2^{\prime}, 7^{\prime}$-dichlorodihydrofluorescein diacetate. Plates were incubated for $20 \mathrm{~min}$ at $37^{\circ} \mathrm{C}$. After incubation and uptake of dye by cells, the dye solution was removed, and cells were again washed twice with warm HBSS to remove all extracellular dye. For DetaNO titration, cells were treated with $100 \mu \mathrm{L}$ of $0 \% \mathrm{~F} 12 \mathrm{~K}$-Se with 0.25 to $5 \mathrm{~m} M$ DetaNO. For vitamin $\mathrm{E}$ analog mitigation of ROS production, cells were treated with $100 \mu \mathrm{L}$ of $0 \%$ F12K -Se medium only as control or containing $1 \mathrm{mM}$ DetaNO, 10 or $50 \mu M$ vitamin E analogs, or methanol vehicle control where appropriate. Medium-only controls were used as the baseline to determine fold-change for treatments of each cell clone. Fluorescence was measured at designated time points on a BioTek Synergy H1 microplate reader using an autogain feature resulting in intra-assay coefficients of variation ranging from 2.32 to $8.28 \%$ with an average of $5.16 \%$. Each plate contained samples and replicates representing a single timepoint and concentration of analogs.

\section{Cellular Damage}

Quantification of lipid membrane peroxidation was conducted using the Abcam Lipid Peroxidation Assay Kit (Cell-based). In short, cells were plated in a 12-well plate and incubated for $18 \mathrm{~h}$, after which the medium was changed with like $10 \%$ F12K + Se medium containing appropriate vitamin $\mathrm{E}$ analogs, methanol vehicle control, or medium alone for $6 \mathrm{~h}$ at $37^{\circ} \mathrm{C}$ in $5 \% \mathrm{CO}_{2}$. After incubation, cells were treated with $10 \mu \mathrm{M}$ of vi-

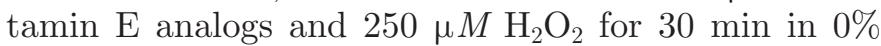
FBS F12K - Se medium. Hydrogen peroxide was used instead of DetaNO for this assay as we were specifically evaluating lipid peroxidation, which is driven more directly by a reactive metabolite such as $\mathrm{H}_{2} \mathrm{O}_{2}$ than by a reactive metabolite-generating compound such as DetaNO. The medium was removed, and an HBSS solution containing assay dye at $1 \times$ was added for incubation at $37^{\circ} \mathrm{C}$ in $5 \% \mathrm{CO}_{2}$ for an additional $30 \mathrm{~min}$. The solution was removed, cells were washed twice with HBSS, trypsinized, pelleted, and resuspended in $200 \mu \mathrm{L}$ of HBSS for analysis by flow cytometry. Lipid peroxidation was determined by shift of fluorescence from red to green, represented by Lipid Peroxidation Index, the red:green relative fluorescent unit ratio, as measured on an Accuri C6 flow cytometer (BD Biosciences).

Apoptosis was quantified by flow cytometry using YoPro dye as an indicator for early apoptotic cells and PI to differentiate necrotic cells. Cells were plated in 6-well tissue-culture plates and allowed to incubate overnight. After $16 \mathrm{~h}$, the medium was removed and replaced with like $10 \%$ F12K +Se medium with or without vitamin $\mathrm{E}$ analogs at 5 or $10 \mu M$ or vehicle control. After $6 \mathrm{~h}$, the medium was again removed and replaced with $0 \% \mathrm{~F} 12 \mathrm{~K}$-Se medium with or without vitamin $\mathrm{E}$ analogs at 5 or $10 \mu M$ or vehicle control and $0.5 \mu M$ DetaNO and incubated at $37^{\circ} \mathrm{C}$ in $5 \% \mathrm{CO}_{2}$ for $24 \mathrm{~h}$. A reduced amount of DetaNO was used for evaluation of apoptosis as this was found to more consistently produce apoptotic cells rather than cell death by other means. After incubation, the medium was removed and collected. Cells were collected by trypsinization and added to previously collected media. Cells were pelleted, resuspended in $1 \mathrm{~mL}$ of ice-cold PBS and transferred to round-bottom polystyrene tubes on ice. A $1 \mu \mathrm{L}$ amount of YoPro dye was added to the appropriate tubes, which were incubated for $20 \mathrm{~min}$ on ice. After incubation, $1 \mu \mathrm{L}$ of PI was added to each tube 2 min before analysis. Cells were analyzed for percentage of cells fluorescing with YoPro and PI on 
an Accuri C6 flow cytometer (BD Biosciences). Cells fluorescing both YoPro and PI dyes were considered to be necrotic, whereas those expressing just YoPro were classified as apoptotic. Control cells not receiving treatment were used as the baseline to present apoptosis as a fold-change compared with these cells.

\section{Endothelial Barrier Integrity}

Analysis of endothelial cell barrier integrity was carried out using the Electrical Cell-Substrate Impedance Sensing system (Applied Biophysics Inc.). Cells were plated in a 96-well collagen-coated plate (96W10idf PET, Applied Biophysics Inc.) with each well containing an electrode comprising $2.09 \mathrm{~mm}^{2}$ of the well surface area. After incubating for approximately $14 \mathrm{~h}$ and resistance having plateaued, the medium was replaced with $10 \%$ F12K +Se medium with or without vitamin $\mathrm{E}$ analogs at a concentration of 5 or $10 \mu M$. After $6 \mathrm{~h}$, the medium was replaced with $1 \%$ F12K -Se medium with vitamin $\mathrm{E}$ analogs at 5 or $10 \mu M$ or vehicle control methanol and DetaNO at $0.5 \mathrm{~m} M$ in addition to a set of wells receiving medium only as control. This assay uses $1 \%$ FBS medium because trials using $0 \%$ experienced losses of cell barrier integrity due to serum starvation alone. Maintaining cells in 1\% FBS medium prevented this barrier loss. A lesser concentration of DetaNO compared with other assays was also used as $0.5 \mathrm{mM}$ produces a more consistent disruption in barrier integrity over time compared with higher concentrations. Additionally, the $5 \mu M$ dose was included for evaluation of barrier integrity and apoptosis after it was discovered that $\gamma$-tocotrienol significantly disrupted the barrier integrity at $10 \mu M$. As such, a lower concentration was evaluated to determine if $\gamma$-tocotrienol may not have such detrimental effects at a lower concentration and if any analogs may still have physiologic activity at this lower concentration. Cell barrier integrity was monitored continuously for $48 \mathrm{~h}$. Parameters for analysis were as follows: single-frequency, 4,000-Hz, 180-s interval time. Resistance was normalized for each individual well to the value of the timepoint before addition of the DetaNO treatment. Resistance curves were used to derive area under the curve (AUC) values.

\section{Statistics}

Statistical analysis was carried out using JMP Pro 14.3.0 (SAS Institute Inc.). Statistics represented with an asterisk, including those for viability assays for assessing vitamin E analogs and DetaNO titration and cytotoxicity of vitamin $\mathrm{E}$ analogs, compared each treatment to a single medium-only control using parametric pairwise comparison with Dunnett's correction for multiple comparisons or nonparametric Steel test. For the cytotoxicity assay, this control is not specifically shown but represented by $0 \%$ cytotoxicity. Differences outlined by differing letters, including the OxiSelect ROS, LDH-based cytotoxicity including DetaNO treatment, lipid peroxidation, cellular apoptosis assays, and endothelial barrier integrity AUC were determined using pairwise Tukey-Kramer honestly significant difference (HSD) with multiple comparisons. For these comparisons, each treatment was compared with each other treatment within a given graph. Single timepoint analysis for assessing endothelial barrier integrity was carried out using a repeated-measures 2-way ANOVA Dunnett's multiple comparison test $(P<0.05)$. This analysis compared each individual timepoint of treatments to the DetaNO-only treatment.

\section{RESULTS}

\section{Cell Viability}

The effect of vitamin $\mathrm{E}$ analog on cell viability varied according to specific analog, dose, and incubation time. Cell viability as assessed by cellular ATP abundance showed no differences among treatments after $6 \mathrm{~h}$ of incubation $(P<0.05$; Figure $2 \mathrm{~A})$. After $24 \mathrm{~h}$ of incubation, $\alpha$-tocopherol increased cell viability $15.7 \pm 1.9 \%$ above control cells treated with medium only, whereas $\gamma$-tocopherol did not affect cellular viability $(P<0.05)$. We found viability of cells treated with a $50 \mu M$ dose of $\gamma$-tocopherol to be reduced by $58.5 \%(P<0.05)$ after $24 \mathrm{~h}$ of treatment (Figure 2B).

Cytotoxicity of analogs was assessed by LDH release after 6 and $24 \mathrm{~h}$ of incubation and presented as a percentage of cytotoxicity where control cells maintained in like base growth medium represented $0 \%$ cytotoxicity and lysed cells represented $100 \%$ cytotoxicity (Figure 2C, D). All 3 analogs showed an increase in cytotoxicity with increasing dosage at both 6 and $24 \mathrm{~h}$, whereas increases due to $\alpha$-tocopherol and $\gamma$-tocopherol, although significant, never increased to $>5 \%(P<0.05)$. Unlike the tocopherols, $\gamma$-tocotrienol caused more severe cytotoxicity at $25 \mu M(10.1 \pm 2.1 \%)$ and $50 \mu M(21.2$ $\pm 0.2 \%)$ doses, greater than that of $\alpha$-tocopherol or $\gamma$-tocopherol $(P<0.05)$.

\section{Reactive Oxygen Species}

Production of ROS was evaluated in BMEC after $30 \mathrm{~min}, 1 \mathrm{~h}$, and $6 \mathrm{~h}$ of treatment with $1 \mathrm{~m} M$ DetaNO, a nitric oxide donor (Figure 3). DetaNO was selected as a pro-oxidant antagonist after evaluating 


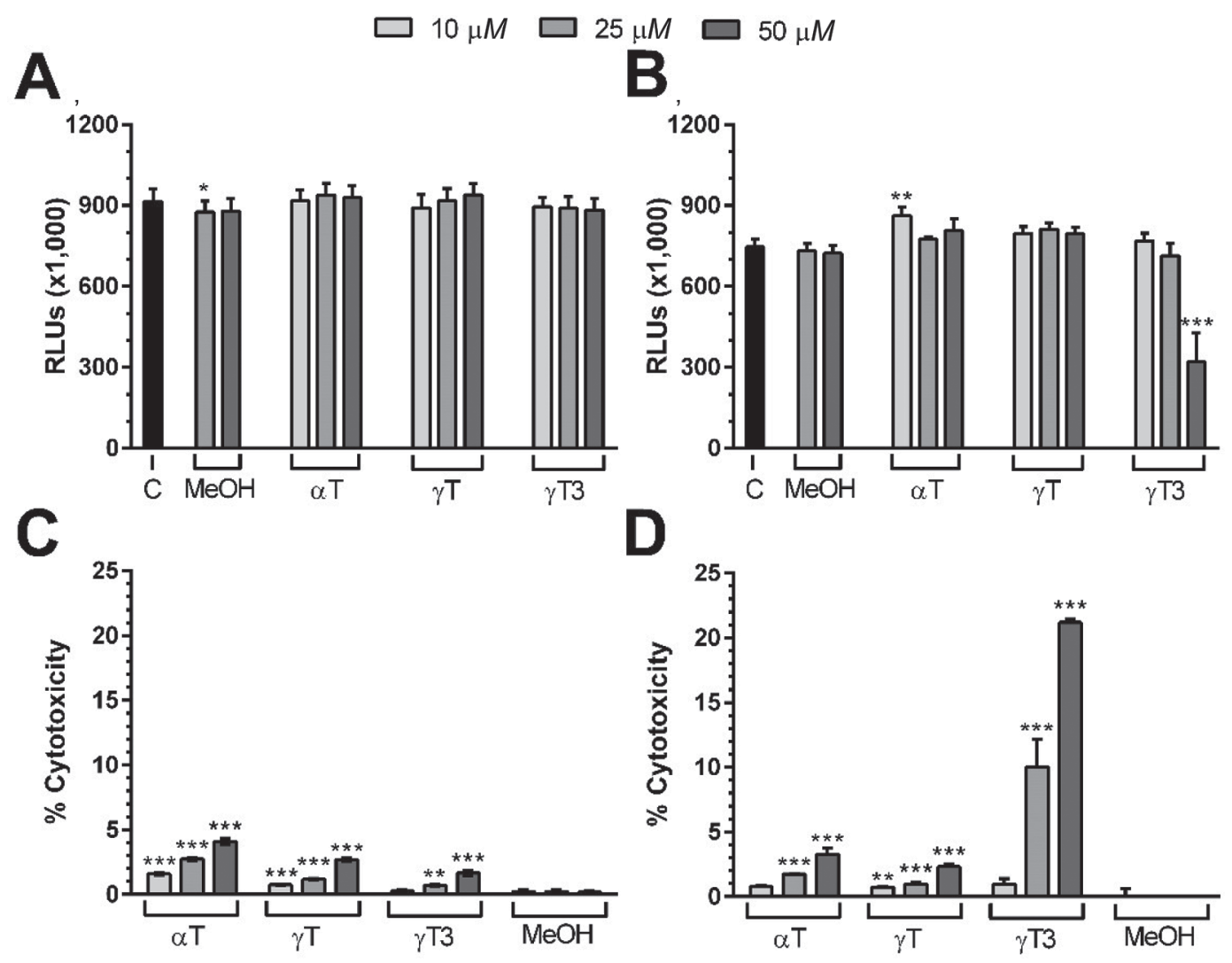

Figure 2. Relative luminescent units (RLU, mean \pm SEM) measuring cell viability by ATP abundance after $6 \mathrm{~h}(\mathrm{~A})$ or $24 \mathrm{~h}(\mathrm{~B})$ in bovine mammary endothelial cells treated with vitamin E analogs $\alpha$-tocopherol $(\alpha \mathrm{T})$, $\gamma$-tocopherol $(\gamma \mathrm{T})$, or $\gamma$-tocotrienol $(\gamma \mathrm{T} 3)$ at differing concentrations or methanol $(\mathrm{MeOH})$ vehicle control. Methanol vehicle was not assessed for $10 \mu M$ treatments. Similarly, cytotoxicity of bovine mammary endothelial cells was measured by cellular lactate dehydrogenase after $6 \mathrm{~h}(\mathrm{C})$ or $24 \mathrm{~h}(\mathrm{D})$ of treatment with $\alpha \mathrm{T}, \gamma \mathrm{T}$, $\gamma \mathrm{T} 3$, or MeOH vehicle control. Percentage cytotoxicity (mean \pm SEM) is determined as the release of lactate dehydrogenase proportional to maximum release from lysed cells, considered to have $100 \%$ cytotoxicity, and untreated control cells, considered to have $0 \%$ cytotoxicity $(\mathrm{n}=4)$. ${ }^{*} P<0.05,{ }^{* *} P<$ $0.01,{ }^{* * *} P<0.001$.

several candidates in multiple bovine cell types for a compound that would generate ROS over time without causing significant viability issues yet stimulate moderate cytotoxicity at higher concentrations. DetaNO was desirable for meeting these criteria, especially its slower development of ROS compared with commonly used compounds, such as 2,2'-azobis(2-amidinopropane) dihydrochloride, in addition to its aqueous solubility, precluding the use of additional vehicle. At a concentration of $1 \mathrm{~m} M$, DetaNO reliably stimulated ROS accumulation without negatively affecting cell viability (Figure 4). Concentrations of 10 and $50 \mu M$ of vitamin $\mathrm{E}$ analogs were used to represent the high end of the physiologic range of $\gamma$-tocopherol and $\alpha$-tocopherol, respectively, in the blood of dairy cattle supplemented with either analog. When using $10 \mu M$ concentrations of analogs, both $\gamma$-tocopherol and $\gamma$-tocotrienol were found to reduce the production of ROS compared with DetaNO antagonist alone $(P<0.05$; Figure 3$)$. When treated with $50 \mu M$, all 3 analogs reduced the production of ROS by DetaNO to a similar amount $(P<$ $0.05)$.

\section{Cellular Damage and Death}

The antagonist DetaNO alone did not produce lipid peroxides as measured by the protocol we used. Instead, $\mathrm{H}_{2} \mathrm{O}_{2}$ was used as a reactive metabolite to quantify potential reductions in lipid peroxide production by vitamin $\mathrm{E}$ analogs (Figure 5). Although $\mathrm{H}_{2} \mathrm{O}_{2}$ did not increase lipid peroxidation statistically, the difference trended toward a difference $(P=0.06)$ as did that between $\mathrm{H}_{2} \mathrm{O}_{2}$ and $10 \mu M \alpha$-tocopherol. Only the $10 \mu M$ $\gamma$-tocopherol treatment reduced the formation of lipid peroxides after a 30-min $\mathrm{H}_{2} \mathrm{O}_{2}$ challenge $(P<0.05)$.

Vitamin E analogs were also evaluated for their ability to limit cellular cytotoxicity after $2 \mathrm{~m} M$ DetaNO treatment, a dose determined to cause cellular 

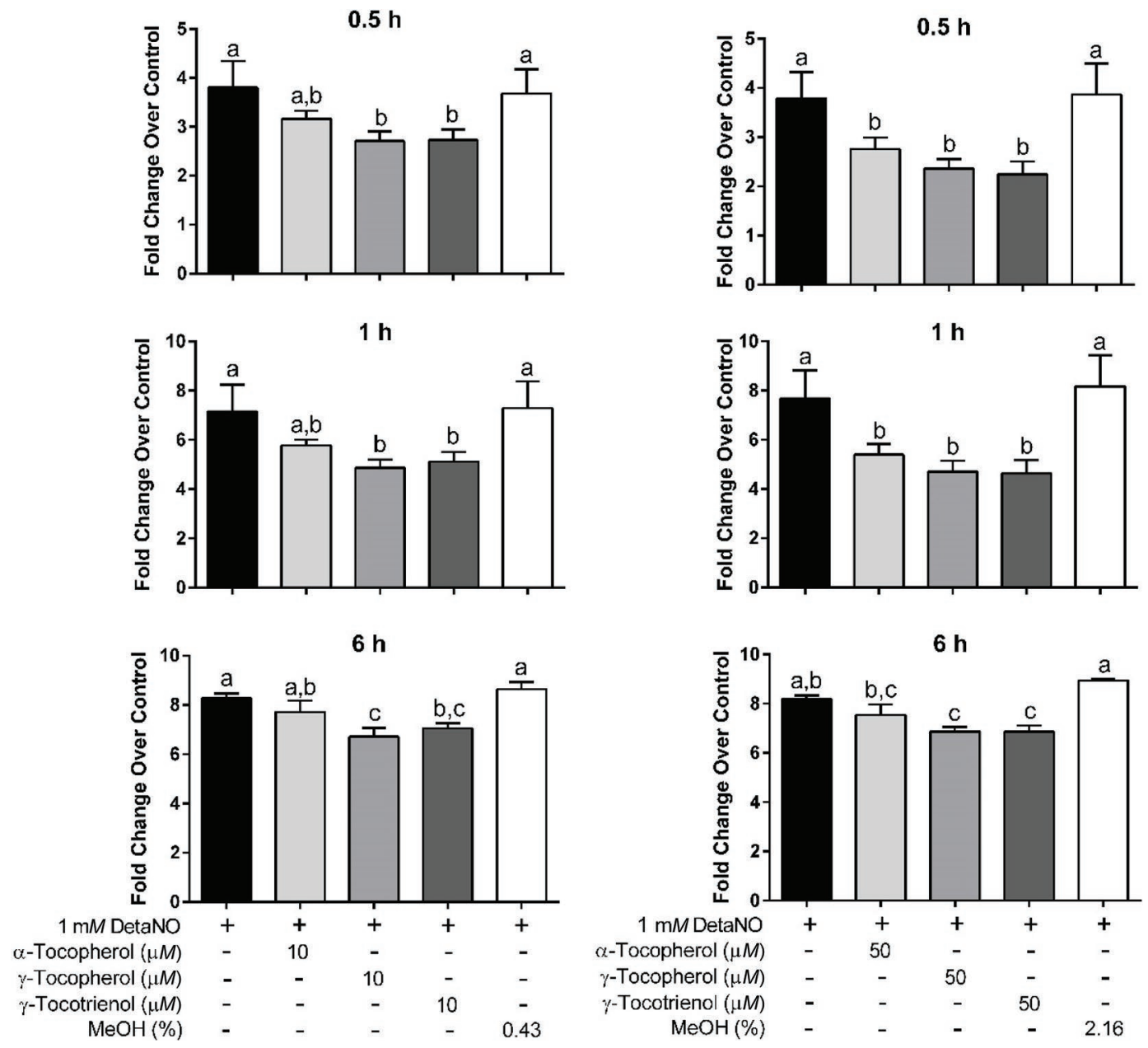

Figure 3. Reactive oxygen species accumulation (mean \pm SEM) in bovine mammary endothelial cells after 0.5-, 1-, or 6-h incubations with $1 \mathrm{~m} M$ DetaNO and either $10 \mu M(\mathrm{n}=6)$ or $50 \mu M(\mathrm{n}=5)$ of vitamin $\mathrm{E}$ analogs or methanol $(\mathrm{MeOH})$ vehicle control. Values are presented as the fold change over untreated control cells incubated in like medium. Cells receiving vitamin E or vehicle control were preincubated with appropriate analog or $\mathrm{MeOH}$ vehicle for $6 \mathrm{~h}$ and treated again with addition of DetaNO. Differing letters represent significant difference between treatments $(P<0.05)$.

cytotoxicity. After a 24-h treatment, $\gamma$-tocopherol and $\gamma$-tocotrienol reduced DetaNO-derived cytotoxicity, and $\gamma$-tocotrienol did so to a greater amount than $\gamma$-tocopherol $(P<0.05$; Figure $6 \mathrm{~A})$. Additionally, when $30 \mu M \alpha$-tocopherol alone was compared with $10 \mu M$ concentrations of each analog mixed together, the analog mixture offered greater protection from cellular damage or death than $\alpha$-tocopherol alone $(P<0.05$; Figure 6B).

In addition to measuring cytotoxicity, early stage apoptosis was evaluated. A $0.5 \mathrm{~m} M$ treatment of DetaNO for $24 \mathrm{~h}$ resulted in a 1.6-fold increase in apoptosis in cells. Unlike the effects of analogs on cellular cytotoxicity, $\gamma$-tocopherol alone reduced the number of cells undergoing apoptosis and did so at both 5 and 10 $\mu M(P<0.05)$. The $5 \mu M$ treatment of $\gamma$-tocopherol nearly reduced apoptosis to values equal to those of control samples, with only a $1.04 \pm 0.41$ fold change over control cells (Figure 7).

\section{Electrical Cell-Substrate Impedance Sensing}

A $0.5 \mathrm{~m} M$ treatment of DetaNO was used to disrupt the endothelial barrier integrity of BMEC. At individual timepoints over $48 \mathrm{~h}, 5 \mu M \gamma$-tocopherol was the only dose-analog combination to limit the damage to barrier integrity inflicted by DetaNO, specifically at 29to 41-h timepoints $(P<0.05$; Figure $8 \mathrm{~A}, \mathrm{~B})$. Although $\alpha$-tocopherol did not have an effect at individual timepoints, AUC analysis showed that for both 5 and $10 \mu M$ treatments, $\alpha$-tocopherol increased the AUC compared with DetaNO alone, representing a greater sustained 
A

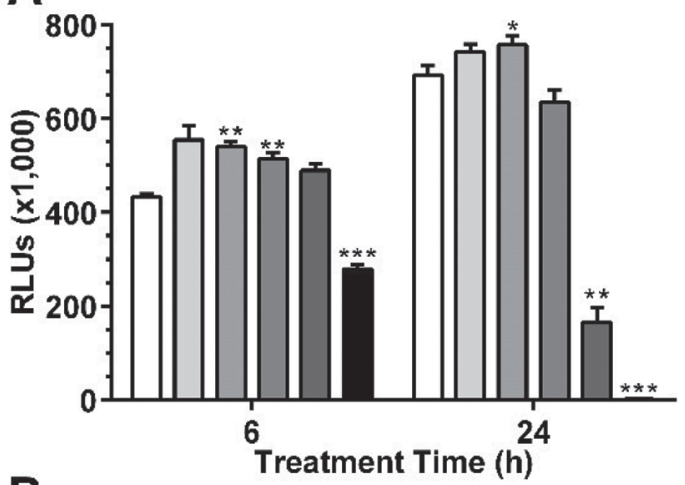

B

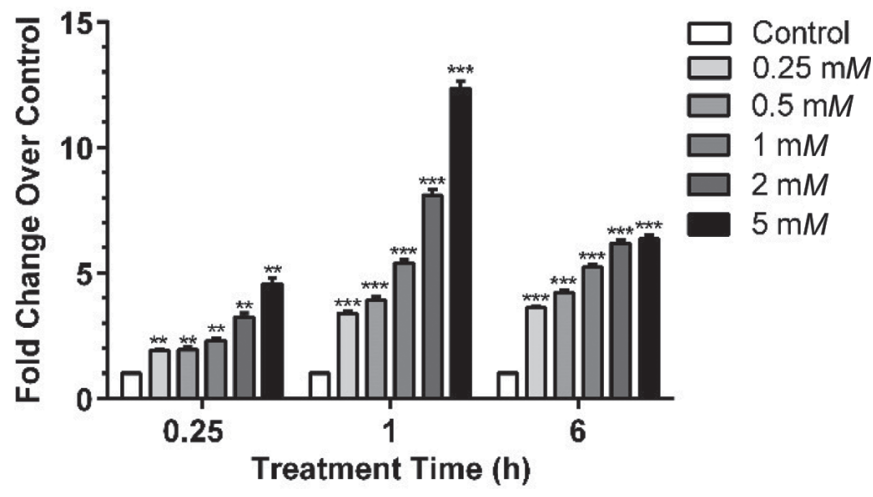

Figure 4. Initial titration of DetaNO on primary bovine mammary endothelial cells to assess potential concentrations for treatment. (A) Relative luminescent units (RLU) representing viability of cells as measured by ATP abundance after treatment with DetaNO concentrations ranging from $0.25 \mathrm{~m} M$ to $5 \mathrm{~m} M$ for $6 \mathrm{~h}$ and $24 \mathrm{~h}$. (B) Reactive oxygen species production within cells, represented as fold change (mean \pm SEM) compared with untreated control cells incubated in like medium, after treatment with concentrations of DetaNO ranging from $0.25 \mathrm{~m} M$ to $5 \mathrm{~m} M$ for $0.25 \mathrm{~h}, 1 \mathrm{~h}$, and $6 \mathrm{~h}(\mathrm{n}=4)$. ${ }^{*} P<0.05$, $* * P<0.01, * * * P<0.001$.

barrier integrity over the 48-h time course $(P<0.05$; Figure 8C, D). Similarly, the AUC of $\gamma$-tocopherol was greater than DetaNO alone for both 5 and $10 \mu M$ doses and did not differ from that of $\alpha$-tocopherol $(P<0.05)$. Surprisingly, $\gamma$-tocotrienol dose dependently reduced the endothelial cell barrier more severely and at a faster rate than the $0.5 \mathrm{~m} M$ DetaNO treatment alone, differing from the DetaNO treatment at time points 14 to $48 \mathrm{~h}$ and 12 to $48 \mathrm{~h}$ for 5 and $10 \mu \mathrm{M}$ analog concentrations, respectively. Such a reduction in barrier integrity by $\gamma$-tocotrienol reduced the AUC for both treatment doses compared with all other treatments $(P<0.05)$.

\section{DISCUSSION}

Through proper management and nutrition, the prevalence of oxidative stress can be reduced, yet there remains a need for interventions to further limit its con- tribution to disease predisposition in transition dairy cows. One such intervention may be the use of non$\alpha$-tocopherol analogs of vitamin E. Non- $\alpha$-tocopherol analogs of vitamin $\mathrm{E}$ have considerably shorter physiological half-lives than $\alpha$-tocopherol, which may reduce the risk for adverse effects potentially associated with greater supplementation of $\alpha$-tocopherol (Bouwstra et al., 2010a,b; Schmolz et al., 2016). In this study, $\gamma$-tocopherol presented antioxidative functions at physiological concentrations in an in vitro primary BMEC oxidant challenge model without evidence of adverse effects on cellular viability. Intriguingly, these effects were found to be more potent than $\alpha$-tocopherol in several instances.

Primary BMEC play a unique role in modeling the interaction between oxidants and vitamin $\mathrm{E}$ analogs. Endothelial cells act as a physical barrier to circulating cells and as regulators of cellular signaling between circulation and underlying tissue (Ryman et al., 2015). With direct exposure to systemic circulation, endothelial cells are exposed to and affected by the systemic oxidant environment that, in addition to other inflammatory signals, contribute to their direct and indirect control over the migration of leukocytes to sites of inflammation (Ryman et al., 2015). Controlling migration of leukocytes as a physical barrier and through inflammatory signaling places endothelial cells at a nexus between oxidative stress and its ultimate nega-

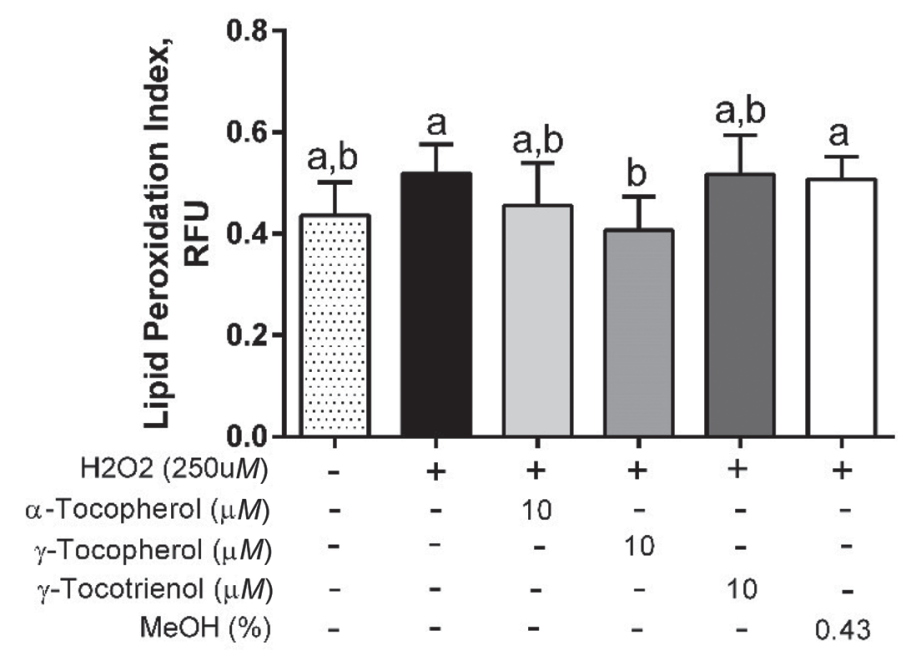

Figure 5. Lipid peroxidation, as represented by a Lipid Peroxidation Index, was determined with flow cytometry by the shift in ratio of green:red fluorescence $[$ mean \pm SEM, relative fluorescence units $(\mathrm{RFU})$ ] where a larger ratio indicates greater lipid peroxidation. Bovine mammary endothelial cells were treated with $10 \mu M$ of appropriate analogs or methanol $(\mathrm{MeOH})$ vehicle control for $6 \mathrm{~h}$ pretreatment and again with analogs or vehicle and $250 \mu M$ hydrogen peroxide $\left(\mathrm{H}_{2} \mathrm{O}_{2}\right)$ for $30 \mathrm{~min}(\mathrm{n}=6)$. Differing letters represent significant difference between treatments $(P<0.05)$. 
tive effects on inflammatory responses. For this reason, maintaining viable and functional endothelial cells is an essential part of effective immune responses.

An additional unique and advantageous characteristic of BMEC to model the effects of vitamin E analogs is a lack of measurable CYP4F2 transcript expression (Kuhn et al., 2020). The cytochrome P450 family 4 subfamily $\mathrm{F}$ member 2 enzyme (CYP4F2), is thought
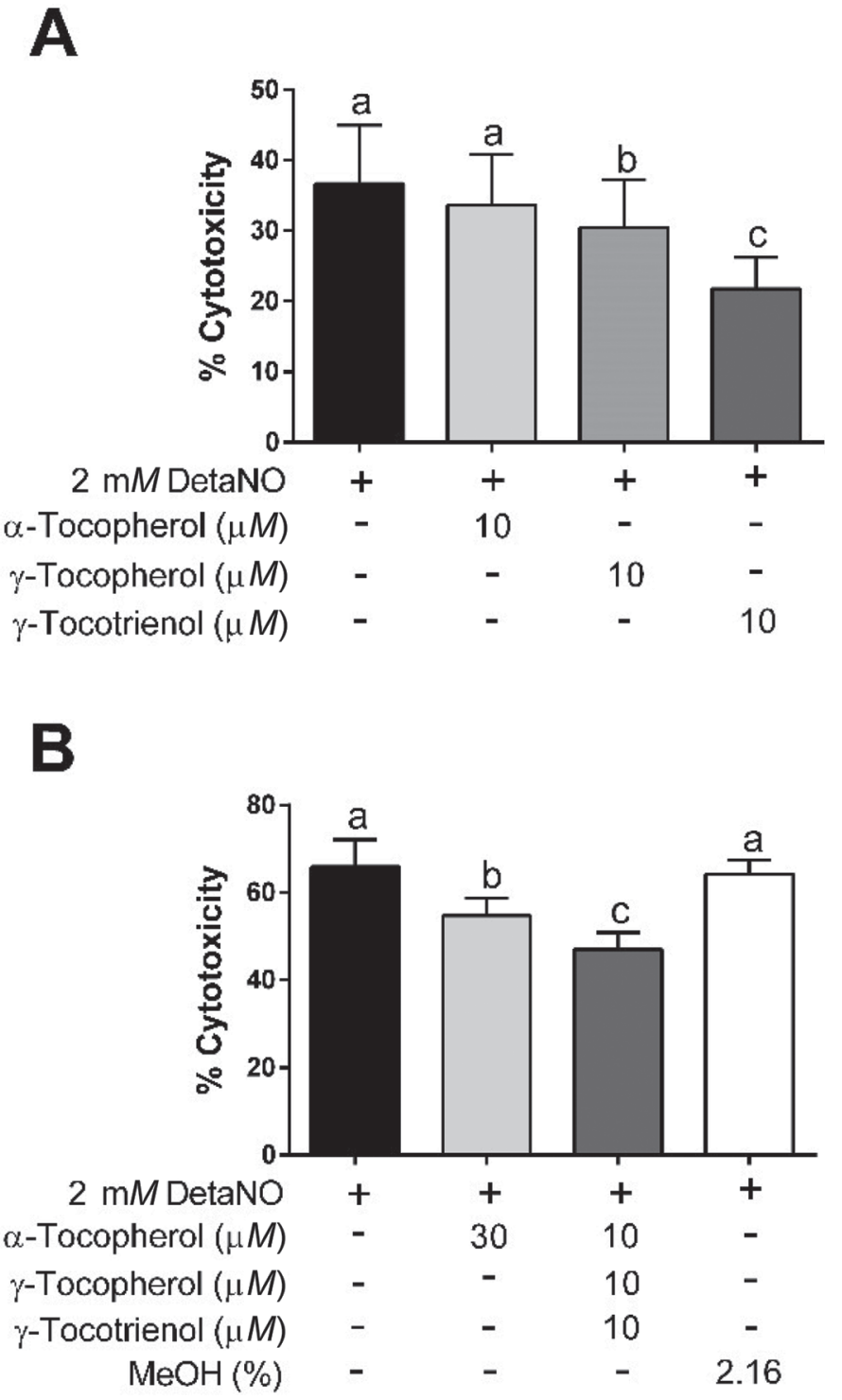

Figure 6. Percentage cytotoxicity (mean \pm SEM) of bovine mammary endothelial cells as measured by lactate dehydrogenase release, relative to lactate dehydrogenase release of untreated control $(0 \% \mathrm{cy}-$ totoxicity) and lysed cells (100\% cytotoxicity), after treatment with 2 $\mathrm{m} M$ DetaNO. Cells treated with vitamin $\mathrm{E}$ analogs were preincubated for $6 \mathrm{~h}$ with analogs before addition of DetaNO for an additional $24 \mathrm{~h}$ incubation. Analogs were either supplemented individually $(\mathrm{A}, \mathrm{n}=10)$ or as a mix $(B, n=4)$. Differing letters represent significant difference between treatments $(P<0.05)$. to be the sole enzyme responsible for the breakdown of vitamin $\mathrm{E}$ analogs and has a preference for metabolizing non- $\alpha$-tocopherol analogs of vitamin $\mathrm{E}$ (Sontag and Parker, 2002, 2007). Without measurable transcript expression of $\mathrm{CYP} 4 \mathrm{~F} 2$, the likelihood that differential metabolism of analogs by CYP4F2 considerably influenced outcome measures is reduced by using a BMEC model. Additionally, downstream metabolites of vitamin $\mathrm{E}$ analogs are known to have biological effects in other species, such as reducing the activity of cyclo-

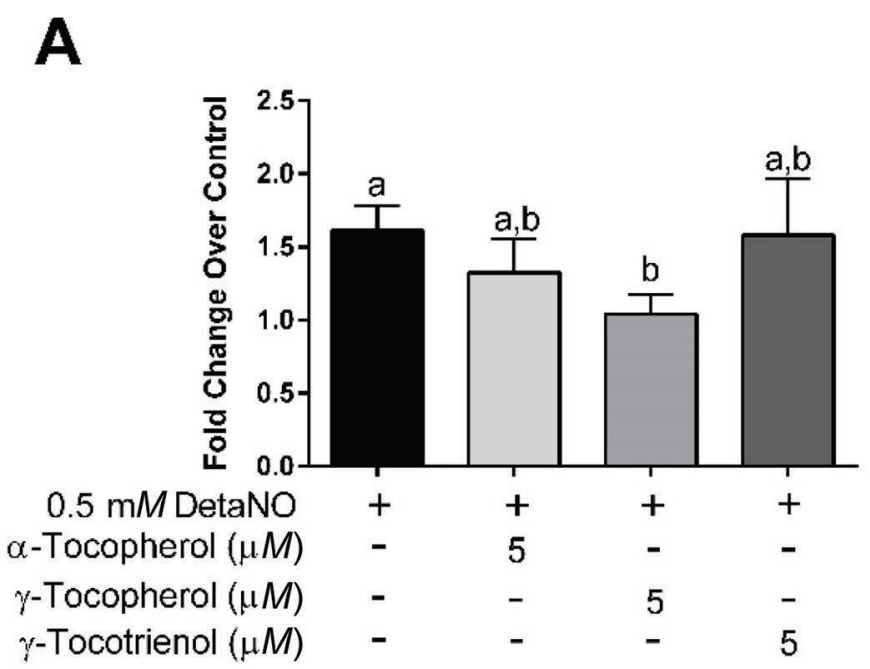

B

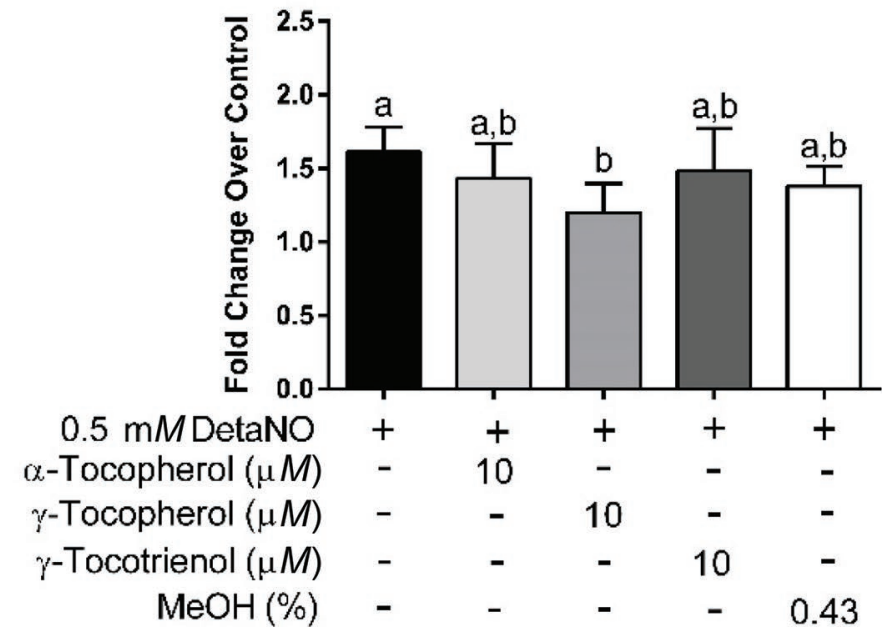

Figure 7. Apoptosis as quantified by flow cytometry using YoPro dye as a marker of apoptosis and exclusion of necrotic cells with propidium iodide dye. Cells treated with vitamin $\mathrm{E}$ analogs or methanol $(\mathrm{MeOH})$ vehicle were preincubated for $6 \mathrm{~h}$ with analogs before addition of DetaNO for an additional $24 \mathrm{~h}$ incubation $(\mathrm{n}=9)$. Values are presented as the fold change (mean \pm SEM) over untreated control cells incubated in like medium. Differing letters represent significant difference between treatments $(P<0.05)$. 
A
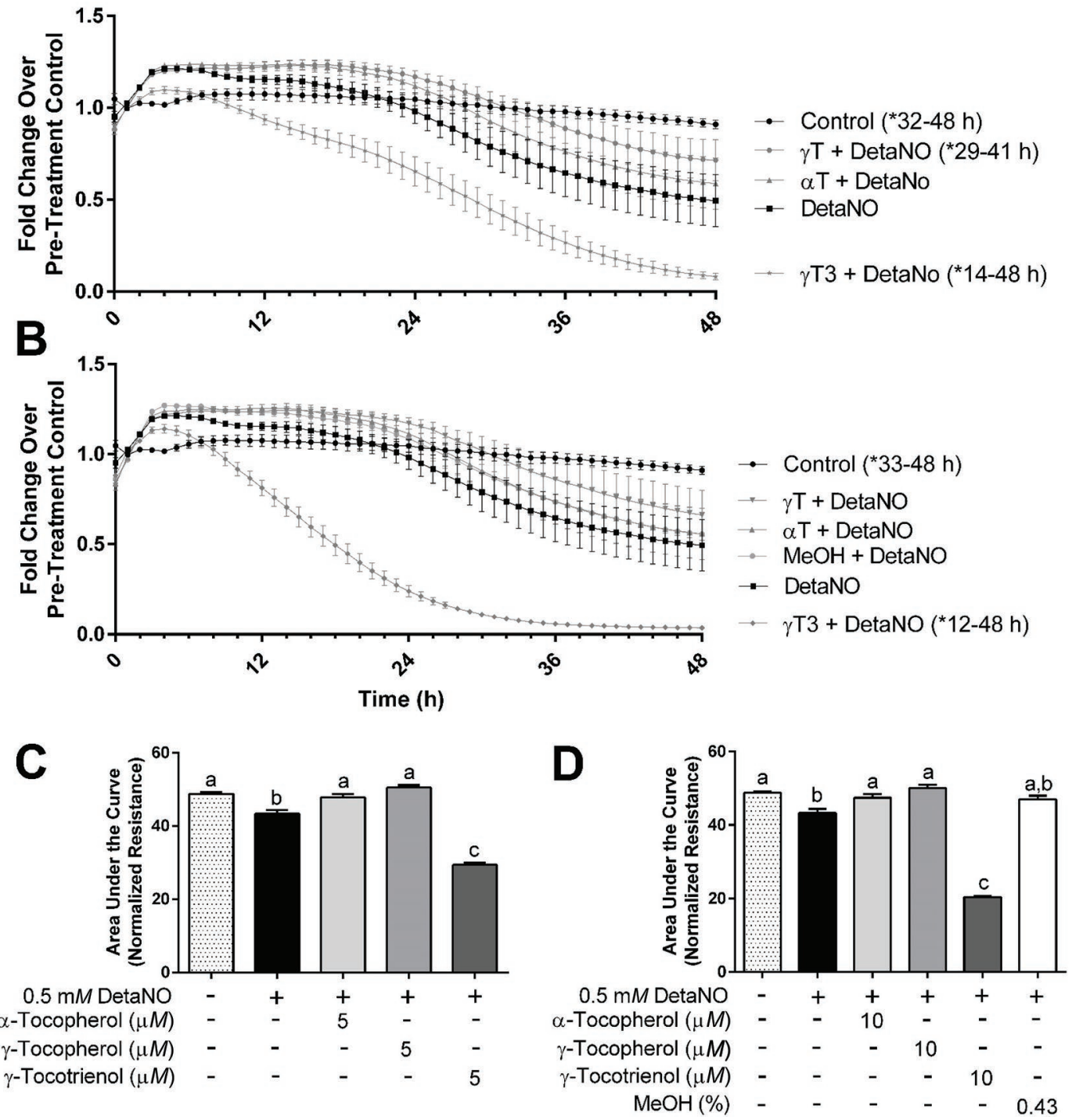

Figure 8. Bovine mammary endothelial cell barrier integrity quantified by electric cell-substrate impedance sensing. Cells were grown to confluency, pretreated for $6 \mathrm{~h}$ with vitamin $\mathrm{E}$ analogs $\alpha$-tocopherol $(\alpha \mathrm{T}), \gamma$-tocopherol $(\gamma \mathrm{T})$, or $\gamma$-tocotrienol $(\gamma \mathrm{T} 3)$ or methanol $(\mathrm{MeOH})$ vehicle and challenged with $0.5 \mathrm{~m} M$ DetaNO $(\mathrm{n}=6)$. Normalized resistance (mean \pm SEM) was measured for analog treatments of $5 \mu M(\mathrm{~A})$ and 10 $\mu M(\mathrm{~B})$ for $48 \mathrm{~h}(\mathrm{~A} / \mathrm{B})$. Asterisk $(*)$ in key represents significant differences between labeled treatment and DetaNO treatment for time period (in hours) listed by 2 -way ANOVA $(P<0.05)$. Area under the curve quantification (mean \pm SEM) was derived for all treatments as measured for $48 \mathrm{~h}$ for analog concentrations $5 \mu M(\mathrm{C})$ and $10 \mu M(\mathrm{D})$. Differing letters represent significant difference between treatments $(P<0.05)$.

oxygenase enzymes (Jiang et al., 2008). Altering the activity of an enzyme such as cyclooxygenase 2, which induces ROS production and several proinflammatory lipid mediators, could have measurable effects on ROS production or cell health independent from functions of the primary vitamin E analogs (Hernanz et al., 2014). Without measurable transcript expression of CYP4F2, the likelihood of these metabolites contributing to outcomes measures is greatly reduced. The converse pitfall of not reliably expressing CYP4F2 in BMEC is the potential for negative effects on cellular viability when supplementing vitamin E analogs. Tocotrienols are metabolized faster than any tocopherol in other mammals and found in cattle at substantially lower concentrations than tocopherols supporting its rapid metabolism (Sontag and Parker, 2007; Sadri et al., 2015). A dearth of $C Y P 4 F 2$ mRNA expression and likely activity may have maintained analogs at greater concentrations in 
our model than would be found in bovine tissues where CYP4F2 is present. This could account, in part, for the cytotoxicity noted for $\gamma$-tocotrienol. A relatively greater cytotoxicity of $\gamma$-tocotrienol is consistent with nonbovine studies that show that although typically found at lower concentrations, tocotrienols are generally more cytotoxic compared with tocopherols when used at similar concentrations (McCormick and Parker, 2004; Yam et al., 2009).

When focusing on the antioxidative capacity of vitamin E analogs, we were surprised to find that $\gamma$-tocopherol and $\gamma$-tocotrienol more potently reduced the accumulation of ROS in BMEC compared with $\alpha$-tocopherol. Even more, the reduction in ROS production by $\gamma$-tocopherol translated into a reduction in lipid peroxidation, yet lipid peroxidation was unaffected by $\gamma$-tocotrienol, suggesting potentially differing antioxidant functions or mechanisms. Finding antioxidative functions at the lower concentration of $10 \mu \mathrm{M}$ and a reduction in apoptotic cells at just $5 \mu M \gamma$-tocopherol is important because $\gamma$-tocopherol ranges from $300 \mathrm{n} M$ to $1 \mu M$ in serum of unsupplemented cattle but has been shown to reach $7.2 \mu M$ when supplemented through the diet, although further increasing supplementation has not yet been reported (Sadri et al., 2015; Qu et al., 2018). Finding benefits to cellular health at concentrations that can indeed be reached by supplementing animals with vitamin $\mathrm{E}$ analogs does not guarantee that these results will translate to a whole-animal model but does support the potential practicality of further evaluating $\gamma$-tocopherol supplementation in vivo.

Although $\alpha$-tocopherol and $\gamma$-tocopherol reduced ROS accumulation when supplemented at $50 \mu \mathrm{M}$, it is difficult to interpret these results compared with the equivalent $\gamma$-tocotrienol treatment when ROS results are put into the context of cell viability. Cytotoxicity from $\alpha$-tocopherol and $\gamma$-tocopherol showed dose-dependent and statistically significant increases; however, values never reached $>5 \%$ and showed no detrimental effect on ATP-based viability measurement. It is thus unlikely that effects of tocopherol analogs influenced the production of ROS through changes in viability. Alternatively, $\gamma$-tocotrienol reduced viability and increased cytotoxicity at higher concentrations after $24 \mathrm{~h}$ of incubation. Although 6-h treatments did not reveal any concerns with cellular viability, it is difficult to rule out the potential contribution of cell death to the reduction of ROS accumulation in cells treated with 50 $\mu M \gamma$-tocotrienol.

The importance of the antioxidant capacity afforded by $\gamma$-tocopherol and $\gamma$-tocotrienol becomes apparent when cellular viability in a pro-oxidant environment is assessed. An important result of oxidative stress is damage to lipids in cell membranes that create peroxidation chain reactions, ultimately leading to cellular death (Su et al., 2019). Breaking these chain reactions is a primary antioxidative function of $\alpha$-tocopherol (Kamal-Eldin and Appelqvist, 1996). For the antioxidative functions of $\gamma$-tocopherol and $\gamma$-tocotrienol to be meaningful in a physiological context, any reduction in ROS accumulation by vitamin $\mathrm{E}$ analogs should reduce oxidant damage to cellular macromolecules in some manner. Indeed, $\gamma$-tocopherol did reduce the formation of lipid peroxides in cells as $\alpha$-tocopherol trended toward a significant reduction. Promisingly, utilizing analogs as a mixture, as is more likely to be fed in vivo, provided greater protection against oxidant cytotoxicity than $\alpha$-tocopherol alone at an equivalent concentration (Figure 6B). Although in vivo non- $\alpha$-tocopherol analogs would be metabolized faster than $\alpha$-tocopherol, this difference in cytotoxicity underscores the potential benefits to including non- $\alpha$-tocopherol analogs in vitamin E supplements.

The apparent greater potency of $\gamma$-tocopherol and $\gamma$-tocotrienol in both reducing ROS accumulation and protecting cellular cytotoxicity compared with $\alpha$-tocopherol raises an additional research question. Although these analogs could simply be provided in addition to current $\alpha$-tocopherol supplements, there is evidence in other species that reducing some supplementation of $\alpha$-tocopherol when supplementing analog mixes may enhance the effectiveness of mixed supplements. Microsomal studies assessing the interactions between vitamin $\mathrm{E}$ analogs show that increasing concentrations of $\alpha$-tocopherol increases the rate of metabolism of non$\alpha$-tocopherol analogs through heterotropic cooperativity, reducing their physiological half-life (Sontag and Parker, 2007). Additionally, the $\alpha$-tocopherol transport protein $(\boldsymbol{\alpha T T P})$, a protein that confers protection from metabolism to any analog bound to it, prefers binding of $\alpha$-tocopherol, reducing its relative metabolism. With relative binding affinity for $\alpha$-tocopherol set at $100 \%$, aTTP binds for $\gamma$-tocotrienol and $\gamma$-tocopherol at only 12 and 9\% respectively (Hosomi et al., 1997). As concentration of $\alpha$-tocopherol increases so too does the displacement of non- $\alpha$-tocopherol analogs from $\alpha$ TTP. For these reasons, knowing that $\gamma$-tocopherol and $\gamma$-tocotrienol may be more potent than $\alpha$-tocopherol, reducing $\alpha$-tocopherol to a certain extent may slow the metabolism of non- $\alpha$-tocopherol analogs and in turn increase their antioxidant efficacy.

A specific outcome from the development of oxidative stress are lipid peroxidation chain reactions in cellular plasma membranes that ultimately result in cellular apoptosis (Catala, 2009). Apoptosis is one mechanism by which oxidative stress contributes to dysfunctional 
inflammatory responses (Ryman et al., 2015). Reducing apoptosis is a functional measure of the capacity of vitamin $\mathrm{E}$ analogs to limit lipid peroxidation chain reactions. $\gamma$-Tocopherol, unlike $\alpha$-tocopherol or $\gamma$-tocotrienol, reduced the percentage of cells undergoing oxidant-induced apoptosis. The capacity of $\gamma$-tocopherol to also reduce the production of lipid peroxides produced by $\mathrm{H}_{2} \mathrm{O}_{2}$ is therefore likely not a coincidence. Although cause and effect are not directly shown, reducing lipid peroxidation is certainly one explanation for how $\gamma$-tocopherol may reduce cellular apoptosis in a pro-oxidant environment.

An increased redox balance can ultimately lead to cellular damage and death when not appropriately managed. A consequence to this damage and death is a reduction in the integrity of the vascular endothelium and its ability to regulate inflammatory responses. Although the endothelial barrier may become slightly less cohesive to facilitate migration of leukocytes during inflammatory events, this is a tightly regulated process that results in only minor reductions in barrier integrity. Sustained or severe weakening of the endothelial barrier disrupts its ability to orchestrate inflammatory responses and is a detrimental outcome of pathogen or oxidative damage (Ryman et al., 2016). The ability of $\alpha$-tocopherol and $\gamma$-tocopherol to slow the deterioration of the endothelial barrier due to oxidative damage is a very promising and applicable outcome from this study. Although the mechanism by which these tocopherols reduced oxidant damage of the endothelial barrier function was not directly determined, $\gamma$-tocopherol's suppression of oxidant-induced apoptosis represents one possible mechanism. These data suggest that in vivo, increased endothelial concentrations of $\gamma$-tocopherol may indeed reduce or slow damage to vascular endothelium from oxidative stress faced by some animals during transition or infection (Mavangira et al., 2016; Kuhn et al., 2017).

We found the rapid deterioration in barrier integrity of cells treated with $\gamma$-tocotrienol surprising and inconsistent with cellular viability and cytotoxicity data. A lack of cell death or cytotoxicity from $10 \mu M$ $\gamma$-tocotrienol after $24 \mathrm{~h}$ suggests that the electrical cell-substrate impedance sensing system is sensitive to causes of cellular stress other than outright cytotoxicity that reduce barrier integrity by an undetermined mechanism. In other species, $\gamma$-tocotrienol has been shown to be potently antiangiogenic and induces apoptosis ( $\mathrm{Li}$ et al., 2011). $\gamma$-Tocotrienol did not induce apoptosis after 24-h treatment as quantified by YoPro dye inclusion, a timepoint after barrier integrity began to diminish; however, in this assay $\gamma$-tocotrienol was supplemented alongside DetaNO and not evaluated independently for potential to cause apoptosis. Additionally, a deterioration of the endothelial barrier integrity does not necessarily require cell death but may represent a reduction in the strength and completeness of cell-to-cell junctions (Gonzalez-Mariscal et al., 2011).

Given the results of this study and the relative toxicity of $\gamma$-tocotrienol noted in other cellular and animal models, caution should be taken when supplementing a vitamin E mix containing tocotrienols (Swift et al., 2014). Certainly, tocotrienols have been used in other species in vivo without adverse effects; however, cattle should be monitored closely until safe baseline values are found (Ikeda et al., 2001; Zhao et al., 2015). Alternatively, tocopherol mixes including oils rich in $\gamma$-tocopherol and $\delta$-tocopherol have already been studied in cattle without measured adverse effects (Elsasser et al., 2013; Qu et al., 2018). Unfortunately, these prior studies did not include well-rounded analyses of redox balance or any direct measure of lipid membrane damage from pro-oxidants.

Although these data provide credence for further evaluation of $\gamma$-tocopherol use in dairy cattle, evidence that $\gamma$-tocotrienol can damage cells provides a warning of the work left to be done. The optimal and toxic concentrations in blood or tissue for analogs of vitamin $\mathrm{E}$ in cattle remains essentially unknown. Measuring both tocopherols and tocotrienols in such oils in future studies will help to establish safe or unsafe baselines for supplementation. Nonetheless, antioxidant activities of vitamin $\mathrm{E}$ analog in dairy cattle deserves greater attention given the positive in vitro results shown herein. Specifically, the capacity of non- $\alpha$-tocopherol analogs to increase the antioxidant capacity and reduce oxidative stress in oxidatively challenged animals should be assessed. Dairy cattle that experience oxidative stress during the transition period face an increased predisposition to disease that can result in lost productivity, increased veterinary costs, and reduced animal welfare. The prospect of increasing the antioxidant capacity of transition animals through non- $\alpha$-tocopherol analogs of vitamin $\mathrm{E}$ may provide a viable means to reduce the incidence of transition cow disorders. These potential benefits make further evaluating non- $\alpha$-tocopherol analogs of vitamin $\mathrm{E}$ and interventions that may increase their concentration or physiological longevity important future research focuses.

\section{ACKNOWLEDGMENTS}

The authors are funded by support from the Agriculture and Food Research Initiative Competitive Grants Program (2017-38420-26759 and 2017-67015-26676) from the USDA National Institute of Food and Ag- 
riculture (NIFA), an endowment from the Matilda $\mathrm{R}$. Wilson Fund (Detroit, MI), and the Michigan Alliance for Animal Agriculture. The authors also thank Cayman Chemical Co. for its support of printing the dissertation to which this work contributed. The authors have not stated any conflicts of interest.

\section{REFERENCES}

Aherne, K. M., M. R. Davis, and L. M. Sordillo. 1995. Isolation and characterization of bovine mammary endothelial cells. Methods Cell Sci. 17:41-46. https://doi.org/10.1007/BF00981884.

Borher, J. R., L. A. Goncalves, and P. E. de Felicio. 2002. $\alpha$ - and $\gamma$-tocopherol levels in Nelore steer blood plasma after a single oral treatment of soybean oil deodorizer distillate (SODD). Meat Sci. 61:301-306. https://doi.org/10.1016/S0309-1740(01)00197-8.

Bouwstra, R. J., M. Nielen, J. R. Newbold, E. H. Jansen, H. F. Jelinek, and T. van Werven. 2010a. Vitamin E supplementation during the dry period in dairy cattle. Part II: Oxidative stress following vitamin E supplementation may increase clinical mastitis incidence postpartum. J. Dairy Sci. 93:5696-5706. https://doi.org/10.3168/ jds.2010-3161.

Bouwstra, R. J., M. Nielen, J. A. Stegeman, P. Dobbelaar, J. R. Newbold, E. H. Jansen, and T. van Werven. 2010b. Vitamin E supplementation during the dry period in dairy cattle. Part I: adverse effect on incidence of mastitis postpartum in a double-blind randomized field trial. J. Dairy Sci. 93:5684-5695. https://doi.org/10 $.3168 / \mathrm{jds} .2010-3159$.

Catalá, A. 2009. Lipid peroxidation of membrane phospholipids generates hydroxy-alkenals and oxidized phospholipids active in physiological and/or pathological conditions. Chem. Phys. Lipids 157:111. https://doi.org/10.1016/j.chemphyslip.2008.09.004.

Celi, P. 2011. Oxidative stress in ruminants. Pages 191-231 in Studies on Veterinary Medicine. L. Mandelker and P. Vajdovich, ed. Humana Press. https://doi.org/10.1007/978-1-61779-071-3_13.

Cooney, R. V., A. A. Franke, P. J. Harwood, V. Hatch-Pigott, L. J. Custer, and L. J. Mordan. 1993. Gamma-tocopherol detoxification of nitrogen dioxide: Superiority to alpha-tocopherol. Proc. Natl. Acad. Sci. USA 90:1771-1775. https://doi.org/10.1073/pnas.90.5 1771 .

Elsasser, T. H., S. Kahl, K. M. Lebold, M. G. Traber, J. Shaffer, C.-J. $\mathrm{Li}$, and S. Block. 2013. Short-term alpha- or gamma-delta-enriched tocopherol oil supplementation differentially affects the expression of proinflammatory mediators: Selective impacts on characteristics of protein tyrosine nitration in vivo. Vet. Sci Dev. 3:20-38. https:/ /doi.org/10.4081/vsd.2013.4703.

González-Mariscal, L., M. Quirós, and M. Díaz-Coránguez. 2011. ZO proteins and redox-dependent processes. Antioxid. Redox Signal. 15:1235-1253. https://doi.org/10.1089/ars.2011.3913.

Hernanz, R., A. M. Briones, M. Salaices, and M. J. Alonso. 2014. New roles for old pathways? A circuitous relationship between reactive oxygen species and cyclo-oxygenase in hypertension. Clin. Sci. (Lond.) 126:111-121. https://doi.org/10.1042/CS20120651.

Hosomi, A., M. Arita, Y. Sato, C. Kiyose, T. Ueda, O. Igarashi, H. Arai, and K. Inoue. 1997. Affinity for $\alpha$-tocopherol transfer protein as a determinant of the biological activities of vitamin $\mathrm{E}$ analogs. FEBS Lett. 409:105-108. https://doi.org/10.1016/S0014 -5793(97)00499-7.

Ikeda, S., K. Toyoshima, and K. Yamashita. 2001. Dietary sesame seeds elevate $\alpha$ - and $\gamma$-tocotrienol concentrations in skin and adipose tissue of rats fed the tocotrienol-rich fraction extracted from palm oil. J. Nutr. 131:2892-2897. https://doi.org/10.1093/jn/131 .11 .2892 .

Jiang, Q. 2014. Natural forms of vitamin E: Metabolism, antioxidant, and anti-inflammatory activities and their role in disease prevention and therapy. Free Radic. Biol. Med. 72:76-90. https://doi .org/10.1016/j.freeradbiomed.2014.03.035.
Jiang, Q., X. Yin, M. A. Lill, M. L. Danielson, H. Freiser, and J. Huang. 2008. Long-chain carboxychromanols, metabolites of vitamin E, are potent inhibitors of cyclooxygenases. Proc. Natl. Acad. Sci. USA 105:20464-20469. https://doi.org/10.1073/pnas .0810962106 .

Kamal-Eldin, A., and L. A. Appelqvist. 1996. The chemistry and antioxidant properties of tocopherols and tocotrienols. Lipids 31:671701. https://doi.org/10.1007/BF02522884.

Kuhn, M. J., V. Mavangira, J. C. Gandy, and L. M. Sordillo. 2018. Production of 15-F2t-isoprostane as an assessment of oxidative stress in dairy cows at different stages of lactation. J. Dairy Sci. 101:9287-9295. https://doi.org/10.3168/jds.2018-14669.

Kuhn, M. J., V. Mavangira, J. C. Gandy, C. Zhang, A. D. Jones, and L. M. Sordillo. 2017. Differences in the oxylipid profiles of bovine milk and plasma at different stages of lactation. J. Agric. Food Chem. 65:4980-4988. https://doi.org/10.1021/acs.jafc.7b01602.

Kuhn, M. J., A. K. Putman, and L. M. Sordillo. 2020. Widespread basal cytochrome P450 expression in extrahepatic bovine tissues and isolated cells. J. Dairy Sci. 103:625-637. https://doi.org/10 .3168/jds.2019-17071.

Li, Y., W. G. Sun, H. K. Liu, G. Y. Qi, Q. Wang, X. R. Sun, B. Q. Chen, and J. R. Liu. 2011. $\gamma$-Tocotrienol inhibits angiogenesis of human umbilical vein endothelial cell induced by cancer cell. J. Nutr. Biochem. 22:1127-1136. https://doi.org/10.1016/j.jnutbio 2010.09.012.

Mavangira, V., M. J. Mangual, J. C. Gandy, and L. M. Sordillo. 2016. $15-\mathrm{F}_{2 \mathrm{t}}$-Isoprostane concentrations and oxidant status in lactating dairy cattle with acute coliform mastitis. J. Vet. Intern. Med. 30:339-347. https://doi.org/10.1111/jvim.13793.

Mavangira, V., and L. M. Sordillo. 2018. Role of lipid mediators in the regulation of oxidative stress and inflammatory responses in dairy cattle. Res. Vet. Sci. 116:4-14. https://doi.org/10.1016/j.rvsc.2017 .08 .002 .

McCormick, C. C., and R. S. Parker. 2004. The cytotoxicity of vitamin $\mathrm{E}$ is both vitamer- and cell-specific and involves a selectable trait. J. Nutr. 134:3335-3342. https://doi.org/10.1093/jn/134.12.3335.

NRC. 2001. Nutrient Requirements of Dairy Cattle. 7 th rev. ed. Natl. Acad. Press. https://doi.org/https://dx.doi.org/10.17226/9825.

Qu, Y., T. H. Elsasser, S. Kahl, M. Garcia, C. M. Scholte, E. E. Connor, G. F. Schroeder, and K. M. Moyes. 2018. The effects of feeding mixed tocopherol oil on whole-blood respiratory burst and neutrophil immunometabolic-related gene expression in lactating dairy cows. J. Dairy Sci. 101:4332-4342. https://doi.org/10.3168/ jds.2017-13902.

Ryman, V. E., N. Packiriswamy, and L. M. Sordillo. 2015. Role of endothelial cells in bovine mammary gland health and disease. Anim. Health Res. Rev. 16:135-149. https://doi.org/10.1017/ S1466252315000158.

Ryman, V. E., N. Packiriswamy, and L. M. Sordillo. 2016. Apoptosis of endothelial cells by 13-HPODE contributes to impairment of endothelial barrier integrity. Mediators Inflamm. 2016:9867138. https:/ /doi.org/10.1155/2016/9867138.

Sadri, H., S. Danicke, U. Meyer, J. Rehage, J. Frank, and H. Sauerwein. 2015. Tocopherols and tocotrienols in serum and liver of dairy cows receiving conjugated linoleic acids or a control fat supplement during early lactation. J. Dairy Sci. 98:7034-7043. https: //doi.org/10.3168/jds.2015-9710.

Schmölz, L., M. Birringer, S. Lorkowski, and M. Wallert. 2016. Complexity of vitamin E metabolism. World J. Biol. Chem. 7:14-43. https://doi.org/10.4331/wjbc.v7.i1.14.

Sontag, T. J., and R. S. Parker. 2002. Cytochrome P450 w-hydroxylase pathway of tocopherol catabolism. Novel mechanism of regulation of vitamin E status. J. Biol. Chem. 277:25290-25296. https://doi .org/10.1074/jbc.M201466200.

Sontag, T. J., and R. S. Parker. 2007. Influence of major structural features of tocopherols and tocotrienols on their w-oxidation by tocopherol- $\omega$-hydroxylase. J. Lipid Res. 48:1090-1098. https://doi .org/10.1194/jlr.M600514-JLR200.

Sordillo, L. M., and V. Mavangira. 2014. The nexus between nutrient metabolism, oxidative stress and inflammation in transition cows. Anim. Prod. Sci. 54:1204-1214. https://doi.org/10.1071/AN14503. 
Su, L. J., J. H. Zhang, H. Gomez, R. Murugan, X. Hong, D. Xu, F. Jiang, and Z. Y. Peng. 2019. Reactive oxygen species-induced lipid peroxidation in apoptosis, autophagy, and ferroptosis. Oxid. Med. Cell. Longev. 2019:1-13. https://doi.org/10.1155/2019/5080843.

Swift, S. N., R. L. Pessu, K. Chakraborty, V. Villa, E. Lombardini, and S. P. Ghosh. 2014. Acute toxicity of subcutaneously administered vitamin $\mathrm{E}$ isomers delta- and gamma-tocotrienol in mice. Int. J. Toxicol. 33:450-458. https://doi.org/10.1177/1091581814554929.

Weiss, W. P. 1998. Requirements of fat-soluble vitamins for dairy cows: a review. J. Dairy Sci. 81:2493-2501. https://doi.org/10 3168/jds.S0022-0302(98)70141-9.

Weiss, W. P. 2017. A 100-year review: From ascorbic acid to zinc-mineral and vitamin nutrition of dairy cows. J. Dairy Sci. 100:1004510060. https://doi.org/10.3168/jds.2017-12935.

Yam, M. L., S. R. Abdul Hafid, H. M. Cheng, and K. Nesaretnam. 2009. Tocotrienols suppress proinflammatory markers and cyclo- oxygenase-2 expression in RAW264.7 macrophages. Lipids 44:787797. https://doi.org/10.1007/s11745-009-3326-2.

Zhao, L., I. Kang, X. Fang, W. Wang, M. A. Lee, R. R. Hollins, M. R. Marshall, and S. Chung. 2015. Gamma-tocotrienol attenuates high-fat diet-induced obesity and insulin resistance by inhibiting adipose inflammation and M1 macrophage recruitment. Int. J. Obes. (Lond.) 39:438-446. https://doi.org/10.1038/ijo.2014.124.

\section{ORCIDS}

M. J. Kuhn (1 https://orcid.org/0000-0002-0119-8325

L. M. Sordillo @ https://orcid.org/0000-0001-8873-3134 